\title{
Biological dose summation of external beam radiotherapy for the whole breast and image-guided high-dose-rate interstitial brachytherapy boost in early-stage breast cancer
}

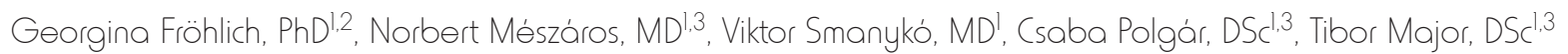 \\ 'Centre of Radiotherapy, National Institute of Oncology, Budapest, Hungary, ${ }^{2}$ Faculty of Natural Sciences, Eötvös Loránd University, Budapest, \\ Hungary, ${ }^{3}$ Department of Oncology, Faculty of Medicine, Semmelweis University, Budapest, Hungary
}

\begin{abstract}
Purpose: To develop an alternative method for summing biologically effective doses of external beam radiotherapy (EBRT) with interstitial high-dose-rate (HDR) brachytherapy (BT) boost in breast cancer. The total doses using EBRT boost were compared with BT boost using our method.

Material and methods: Twenty-four EBRT plus interstitial HDR-BT plans were selected, and additional plans using EBRT boost were created. The prescribed dose was 2.67/40.05 Gy to whole breast and 4.75/14.25 Gy BT or 2.67/10.7 Gy EBRT to planning target volume (PTV) boost. EBRT and BT computed tomography (CT) were registered twice, including fitting the target volumes and using the lung, and the most exposed volume of critical organs in BT were identified on EBRT CT images. The minimal dose of these from EBRT was summed with their BT dose, and these EQD2 doses were compared using BT vs. EBRT boost. This method was compared with uniform dose conception (UDC).

Results: $\mathrm{D}_{90}$ of PTV boost was significantly higher with BT than with EBRT boost: 67.1 Gy vs. 56.7 Gy, $p=0.0001$. There was no significant difference in the dose of non-target and contralateral breast using BT and EBRT boost. $\mathrm{D}_{1}$ to skin, lung, and $\mathrm{D}_{0.1}$ to heart were 58.6 Gy vs. $66.7 \mathrm{~Gy}(p=0.0025), 32.6 \mathrm{~Gy}$ vs. $50.6 \mathrm{~Gy}(p=0.0002)$, and $52.2 \mathrm{~Gy}$ vs. $58.1 \mathrm{~Gy}$ $(p=0.0009)$, respectively, while $\mathrm{D}_{0.1}$ to ribs was $44.3 \mathrm{~Gy}$ vs. $37.7 \mathrm{~Gy}(p=0.0062)$. UDC overestimated $\mathrm{D}_{1}$ (lung) by $54 \%$ $(p=0.0001)$ and $\mathrm{D}_{1}$ (ribs) by $28 \%(p=0.0003)$.

Conclusions: Based on our biological dose summation method, the total dose of PTV in the breast is higher using BT boost than with EBRT. BT boost yields lower skin, lung, and heart doses, but higher dose to ribs. UDC overestimates lung and ribs doses.

J Contemp Brachytherapy 2020; 12, 5: 462-469 DOI: https://doi.org/10.5114/jcb.2020.100379
\end{abstract}

Key words: breast cancer, dose summation, integrated biological doses, boost, interstitial brachytherapy.

\section{Purpose}

The standard of care in curative treatment of earlystage breast cancer is breast-conserving surgery and post-operative external beam radiotherapy (EBRT) to the whole breast $[1,2,3]$. Since $67-100 \%$ of ipsilateral breast recurrences originate from the vicinity of primary tumor site, dose escalation to the tumor bed plays an essential role in the post-operative treatment [4]. Several randomized trials have confirmed that a local boost after whole breast irradiation significantly decreased the rate of local recurrences $[4,5,6,7]$. The most frequently used radiotherapy combination is the whole breast EBRT, with two tangential photon beams and image-guided interstitial brachytherapy (BT), or EBRT boost to the tumor bed [4,5,
$6,7,8,9,10,11,12,13,14,15,16,17]$. This complex combined treatment requires a reliable reporting of dose received by the whole breast, planning target volume (PTV) boost, and critical structures.

Modern high-dose-rate (HDR) interstitial BT boost approach results similarly or even more favorable to local control rate than conventional EBRT boost. Moreover, BT boost has been linked with lower incidence of late side effects [18,19], and the dose of the most exposed part of organs at risk (OARs) correlates with normal tissue toxicity [20].

To report dose-volume parameters properly, overall volumetric doses from external beam- and brachytherapy must be integrated. Since a simple physical dose summation does not take into consideration the different 
biological effects, the equivalent dose given in 2 Gy fractions $\left(\mathrm{EQD}_{2}\right)$ has to be calculated $[21,22]$. The dose distribution of EBRT is assumed to be completely uniform, so the whole breast and nearest OARs included in the fields receive the entire prescribed dose. Subsequently, this equivalent uniform dose is calculated for dose summation with BT doses (uniform dose conception - UDC) [23]. On the other hand, this assumption can be accurate only for organs, which are in the used tangential fields. It is well-known that the most exposed part of OARs in the integrated plans is located in the same region, which receives the largest dose from BT boost. Nevertheless, this 1 or $0.1 \mathrm{~cm}^{3}$ volumes are not always in the same location as the most exposed volume of EBRT [24]. So, simple DVH addition sums the dose of two different volumes.

In previous investigations, authors did not consider the real biological dose of PTV and OARs in combined EBRT with BT or EBRT boost treatments. Terheyden et al. [25] used the above-mentioned UDC method to estimate the doses from EBRT and applied relative physical BT doses only. Shahbazian et al. [26] compared interstitial BT vs. EBRT using photon and electron beams for tumor bed boost in deeply seated tumors. However, they calculated only the relative dose of boost treatments, and they did not consider the total dose of combined therapy. There is no other study in the literature available on the biological summation of dose in combined radiotherapy in earlystage breast cancer.

In the effort to calculate the total biological dose of combined EBRT and BT boost, application of a linear-quadratic formula for a dose-volume parameter is not correct, because $\mathrm{EQD}_{2}$ dose of a voxel is based on $\alpha / \beta$ value and physical dose in the given voxel. In this way, the quadratic behavior of biological dose cannot be taken into consideration. The biological dose has to be calculated voxel-byvoxel in the same organ, but currently, this feature is not available in any of the treatment planning systems.

In the future, the deformable image registration (DIR) could be an appropriate method to integrate EBRT and BT doses both for the boost PTV and for OARs, but at present, it results in significant errors, especially where dose summation is sensitive due to high-dose gradient of BT. Beside different breast and lung anatomy, the main difficulties present plastic catheters in situ, which are not on EBRT image data sets.

We have developed an alternative dose summation method in combined radiotherapy of cervical and prostate cancer $[27,28]$. The aim of the present study was to develop an alternative method for summing the biologically effective doses of whole breast EBRT with interstitial HDR-BT boost in breast cancer, and to compare the results with UDC method. Additionally, the $\mathrm{EQD}_{2}$ total doses of EBRT for the whole breast plus HDR-BT or EBRT boost were also compared using our dose summation method.

\section{Material and methods}

\section{External beam radiotherapy}

Twenty-four EBRT for the whole breast plus interstitial HDR-BT boost plans of recently treated patients with early-stage breast cancer were included in this study. EBRT was performed in supine position, the patients were immobilized with an arm support system. The 40.05 Gy dose was delivered with two tangential $6 \mathrm{MV}$ photon beams, with 2.67 Gy daily fractions using True Beam linear accelerator (Varian Medical Systems, Palo Alto, USA). The dose was prescribed to $95 \%$ of the dose in isocenter. The isocenter was located on the central axis computed tomography (CT) slice, in a midpoint between lung-chest wall interface and skin surface. Field-in-field technique was used to avoid dose heterogeneities in the breast. Eclipse v13.7 (Varian Medical Systems, Palo Alto, USA) treatment planning system was used. Based on our local IGRT protocol, CBCT verification was made before the first three fractions; then, the systematic error was calculated and corrected before fourth fraction, followed by weekly verification. For patients, in whom BT was not achievable, EBRT boost was performed using a uniform clinical target volume (CTV) à PTV expansion margin of $0.5 \mathrm{~cm}$. Therefore, during treatment planning, an additional EBRT boost plan was created using two field-infield conformal beams, where 10.7 Gy was prescribed to the PTV in 2.67 Gy daily fractions, according to the recent recommendations [29].

\section{Brachytherapy}

EBRT to whole breast was complemented with CT-guided interstitial multicatheter HDR-BT boost, 2 to 3 weeks after completing EBRT. The patients were treated with an ${ }^{192}$ Ir source with $370 \mathrm{GBq}$ initial activity, using afterloading technique. The implantations were applied under local anesthesia. Pre-implant CT simulation was performed with a template on the breast to define the PTV according to surgical clips in the tumor bed and needle placement planning. The PTV (equal to the CTV) was defined as the excision cavity, with a margin of 1 to $2 \mathrm{~cm}$ according to the surgical tumor-free margin in all main six directions (contouring protocol was the same as for EBRT boost). Following pre-implant simulation, 9 to 22 plastic needles (median, 16) were inserted into the previously targeted area in a triangular setting using template guidance. Subsequently, a post-implant CT scanning was made for planning purpose using the same Thorax-Mamma Hounsfield Unit set as in EBRT CT scan with $3 \mathrm{~mm}$ slice thickness. Active lengths in the catheters were selected in such a way that the extreme source dwell positions in each catheter were on or close to the PTV surface. HIPO (hybrid inverse planning optimization) method (Oncentra Brachy v.4.5.3, Elekta Brachytherapy, Veenendaal, The Netherlands) was applied to achieve optimal dose distribution, where the reference dose of target volume coverage was at least $90 \%$, keeping dose non-uniformity ratio (DNR) less than 0.35. The dosimetric assumptions in the HIPO were as follow: $100 \%$ minimal (weight, 75 ) and $150 \%$ of maximal dose (weight, 25) to the CTV, 50\% of maximal dose (weight, 40) to the skin, 50\% of maximal dose (weight, 30) to the ribs, and $120 \%$ of maximal dose (weight, 5) to the normal tissue. The prescribed dose was $14.25 \mathrm{~Gy}$ to the PTV in 3 fractions (MicroSelectron v.3 afterloader, Elekta Brachytherapy, Veenendaal, The Netherlands). The de- 
tailed description of our treatment method can be found in previous publications $[30,31,32,33,34]$. The total treatment time of EBRT and BT was 4 weeks (25-28 days). In clinical routine, the UDC method was used to determine the dose constraints for PTV boost and OARs in BT implant, and to calculate their total doses.

\section{Dose summation}

First, the treatment planning CT for EBRT was registered with post-implant CT set of BTs in EBRT treatment planning system in every case. During manual registration, the EBRT CT set was shifted and rotated to match the CTVs of BT and EBRT plans (Figure 1A). Then, another registration was made matching the lungs and ribs of BT and EBRT plans (Figure 1B), when the first registration was not appropriate for these OARs too.

Subsequently, the localization of the most exposed part of OARs in the sum of EBRT and BT plans was evalu- ated. Based on the assessment of dose distributions of the whole breast EBRT and BT boost treatments (Figure 2A), the most exposed part of the skin, ipsilateral lung, and ribs was in a region, where the maximum dose was in BT. So, the BT dose of the most exposed $1\left(D_{1}\right)$ and $0.1 \mathrm{~cm}^{3}$ $\left(\mathrm{D}_{0.1}\right)$ from BT were visualized in EBRT CTs, and an intersection of this isodose volumes and the given organ was created (Figure 2B). The minimal dose of this intersection was calculated in EBRT plans and summated with the dose of this volumes from BT, using a linear-quadratic radiobiological model. In a case of contralateral breast and heart, the most exposed part was in a region, where the maximum dose was in EBRT, as the dose contribution from EBRT part was higher than the dose from BT boost. For these organs, the most exposed 1 and $0.1 \mathrm{~cm}^{3}$ from EBRT were used with the same way. The $\alpha / \beta$ of breast tumor was assumed 4 Gy [29], while for OARs, 3 Gy was used. The minimum dose delivered to $90 \%$ of PTV boost
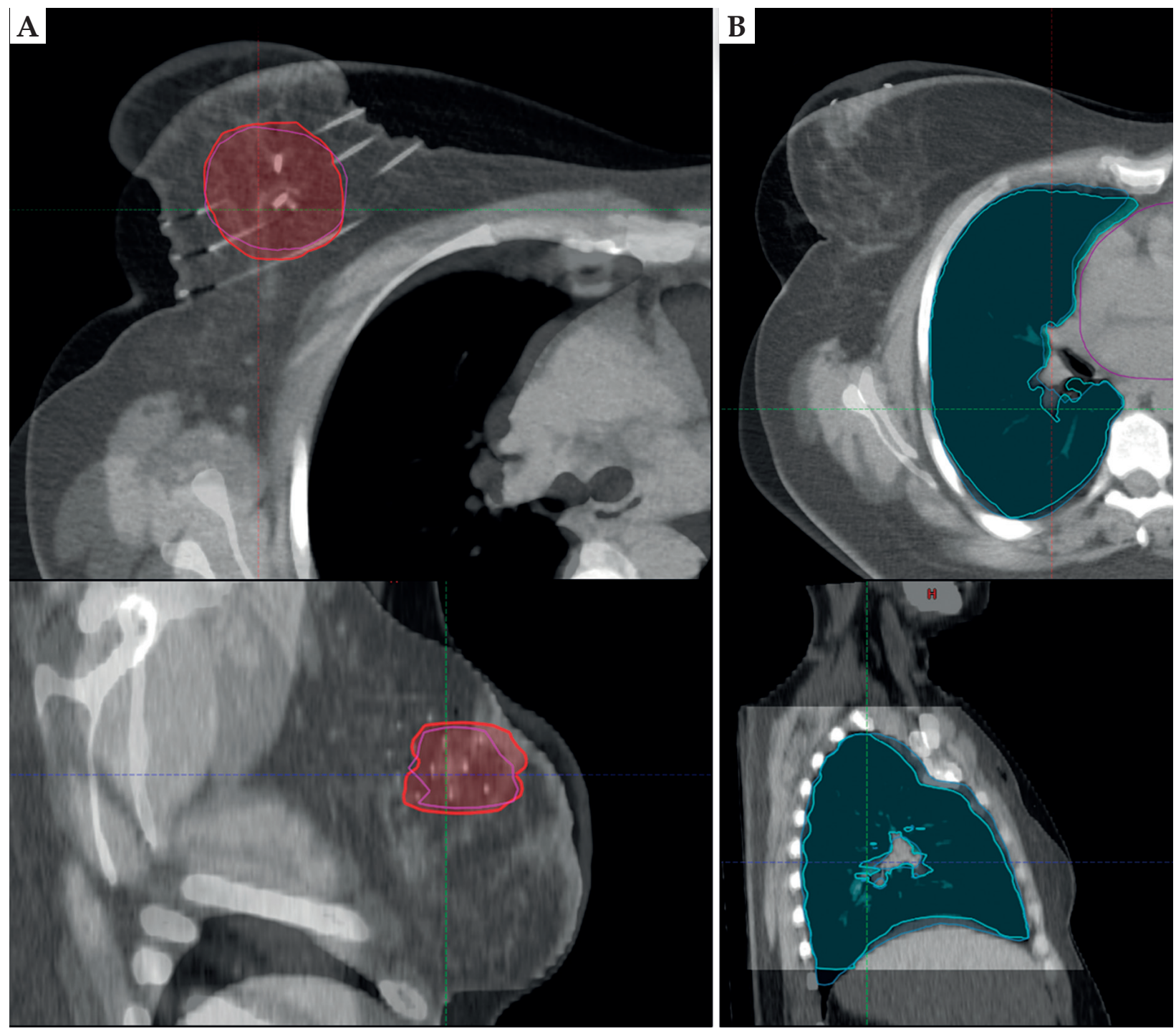

Fig. 1. Registration of the EBRT and BT CT sets based on CTVs (red and pink) (A) and the lung contours (turquoise and blue) (B) on an axial (top) and a sagittal (bottom) plane 

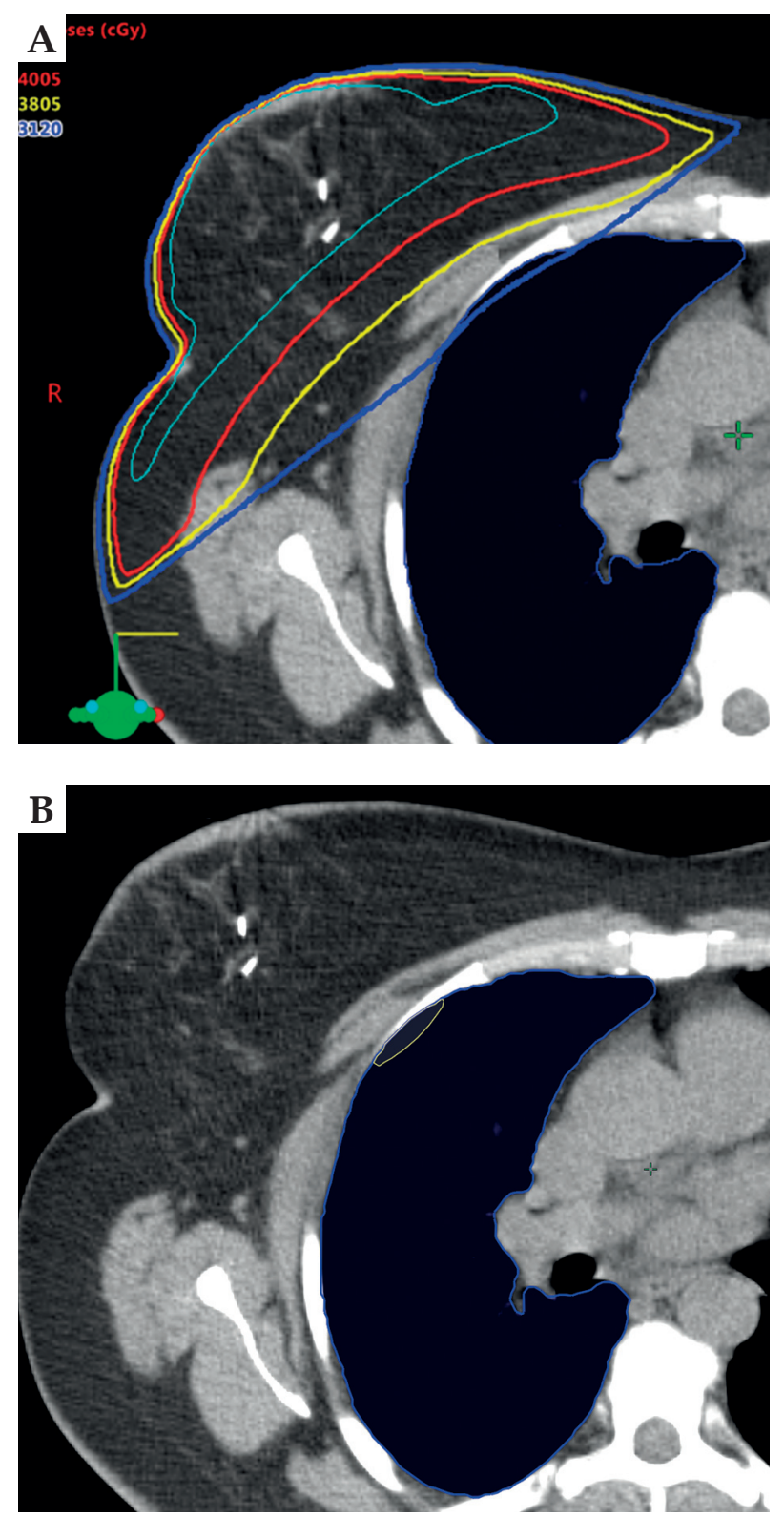

$\left(\mathrm{D}_{90}\right)$ was calculated in EBRT and BT plans, and these doses were summed using a linear-quadratic model.

Wilcoxon-matched pairs test (Statistica 12.5, StatSoft, Tulsa, OK, USA) was used to compare biological total doses of a combination of the whole breast EBRT and BT or EBRT boost in the treatment of early-stage breast tumor. The comparison of our biological dose summation (BDS) and conventional UDC method was also performed with this statistical test.

\section{Results}

\section{EBRT with BT boost}

The mean volume of CTV boost was $47.9 \mathrm{~cm}^{3}(14.3-$ $85.1 \mathrm{~cm}^{3}$ ) in BT. The ratio of CTV boost and the whole breast volume was $0.09(0.03-0.21)$. Nine patients had a tumor in the left breast, and 11 patients in the right one.

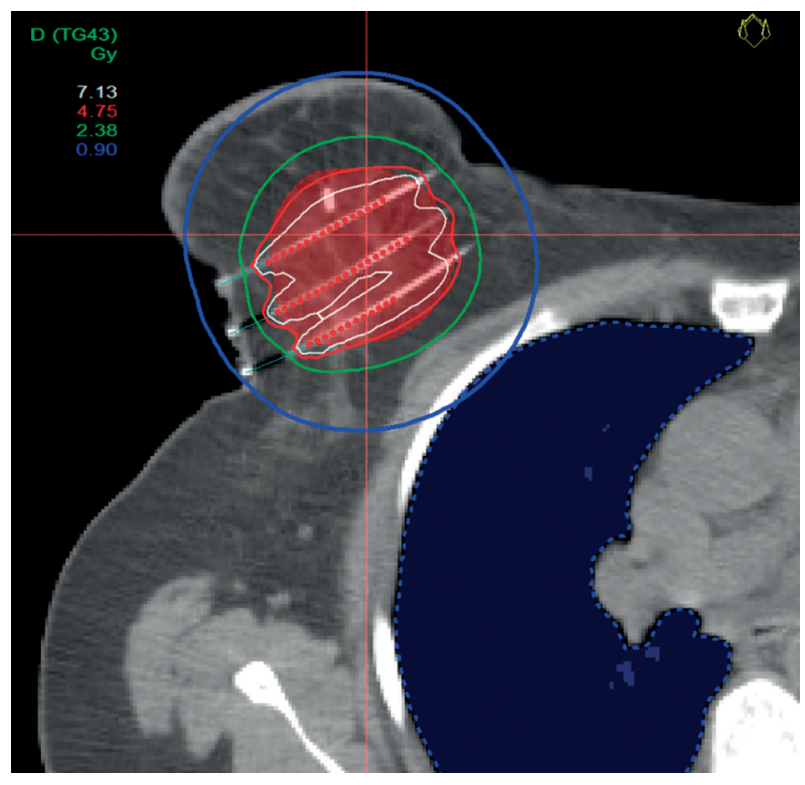

Fig. 2. A) Typical dose distribution of whole breast EBRT (left) and BT boost (right) in the axial slice where the most exposed $1 \mathrm{~cm}^{3}$ part of the lung (blue) is (CTV: red). B) The most exposed $1 \mathrm{~cm}^{3}$ part (yellow) of the lung (blue) in an axial slice of the EBRT CT
We found that $\mathrm{EQD}_{2} \mathrm{D}_{90}$ of PTV boost was $67.1 \mathrm{~Gy}$ (64.973.7 Gy) using EBRT for the whole breast and BT boost. The EQD ${ }_{2}$ mean dose of non-target breast was $45.5 \mathrm{~Gy}$ (45.4-45.6 Gy) on average. The $\mathrm{D}_{1}$ and $\mathrm{D}_{0.1}$ of contralateral breast were 0.72 Gy (0.4-1.0 Gy) and 0.99 Gy (0.6-1.5 Gy), respectively. The $\mathrm{D}_{1}$ and $\mathrm{D}_{0.1}$ of skin were $58.6 \mathrm{~Gy}$ (47.279.9 Gy) and 65.8 Gy (49.2-85.6 Gy), respectively. The $\mathrm{D}_{1}$ and $\mathrm{D}_{0.1}$ of lung were $32.6 \mathrm{~Gy}(15.7-46.2 \mathrm{~Gy})$ and $35.3 \mathrm{~Gy}$ (17.2-48.5 Gy), respectively. The $\mathrm{D}_{1}$ and $\mathrm{D}_{0.1}$ of heart were 50.6 Gy (37.6-61.7 Gy) and 52.2 Gy (38.4-64.0 Gy), respectively, and the $\mathrm{D}_{1}$ and $\mathrm{D}_{0.1}$ of ribs were $40.2 \mathrm{~Gy}$ (34.148.1 Gy) and 44.3 Gy (40.0-53.0 Gy), respectively.

\section{EBRT with EBRT boost}

In EBRT boost, the volume of PTV was larger than in BT, it was $85.3 \mathrm{~cm}^{3}$ on average (range, $35.8-132.5 \mathrm{~cm}^{3}$ ); however, the CTV volume was practically the same, 
$48.2 \mathrm{~cm}^{3}\left(15.2-85.9 \mathrm{~cm}^{3}\right)$, and $47.9 \mathrm{~cm}^{3}\left(14.3-85.1 \mathrm{~cm}^{3}\right)$ in EBRT and BT boost plans $(p=0.1419)$. In comparison of BT and EBRT boost techniques, $\mathrm{D}_{90}$ of PTV boost was significantly higher with BT than with EBRT: 67.1 Gy

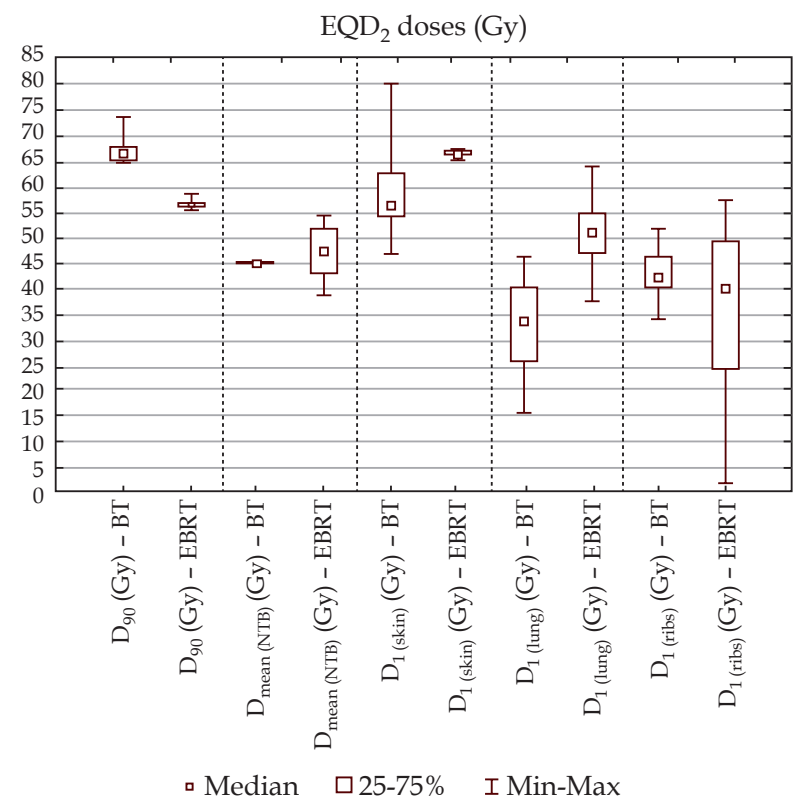

Fig. 3. EQD 2 total doses of external beam radiation therapy plus interstitial HDR-BT boost (BT) and external beam radiation therapy plus external beam radiation therapy boost (EBRT)

$D_{90}$ - the minimum dose delivered to $90 \%$ of PTV boost (Gy), $D_{\text {mean }}$ (NTB) - the mean dose of non-target breast, $D_{1}(x)$ - the minimal dose of the most exposed $1 \mathrm{~cm}^{3}$ of ' $x$ ' organ at risk, where $x$ are skin, lung and ribs vs. $56.7 \mathrm{~Gy}, p=0.0001$. There was no significant difference in the dose of non-target and contralateral breast using BT and EBRT boosts. The $\mathrm{D}_{1}$ to skin was $58.6 \mathrm{~Gy}$ (47.2-79.9 Gy) and 66.7 Gy (65.5-67.5 Gy), $p=0.0025$; the $\mathrm{D}_{1}$ to lung was 32.6 Gy (15.7-46.2 Gy) and 50.6 Gy (37.664.0), $p=0.0002 ; \mathrm{D}_{0.1}$ to heart was 52.2 Gy (38.4-64.0 Gy) and 58.1 Gy (51.7-69.1 Gy), $p=0.0009$, and $\mathrm{D}_{0.1}$ to ribs was 44.3 Gy (40.0-53.0 Gy) and 37.7 Gy (26.6-60.5 Gy), $p=0.0062$ (Figure 3). The detailed results are presented in Table 1.

\section{UDC method}

Comparing our dose summation method to the conventional UDC in the case of combined EBRT with BT boost, we found that the UDC overestimated $\mathrm{D}_{1}$ of lung by $54 \%(p=0.0001)$ and $\mathrm{D}_{1}$ of ribs by $28 \%(p=0.0003)$. The detailed results are shown in Table 2.

\section{Discussion}

Dose escalation has a fundamental role in the post-operative radiotherapy of early-stage breast cancer [4]. Presently, one of the best alternatives for boost is BT; however, a controversy still exists regarding the optimal technique. Traditionally, EBRT with electron or photon beams have been used to deliver the boost dose to the tumor bed [3]. Later, HDR-BT has been also accepted as a safe alternative boost modality $[4,5,6,7,8,9,10,11,12,13,14,15,16,17]$.

Poortmans et al. [18] observed a favorable local control rate with BT boost compared to EBRT boost. They also showed a lower incidence of side effects with BT boost [18], which we confirmed in a previous study [19]. We also demonstrated the correlation between dosevolume parameters and side effects [20]. The volume of

Table 1. $E Q D_{2}$ total doses of external beam radiation therapy plus interstitial HDR-BT boost (EBRT + BT boost) and external beam radiation therapy plus external beam radiation therapy boost (EBRT + EBRT boost)

\begin{tabular}{lccc}
\hline$E_{2}$ & EBRT + BT boost & EBRT + EBRT boost & $p$-value ${ }^{* *}$ \\
\hline$D_{90}$ (Gy) & $67.1(64.9-73.7)$ & $56.7(55.3-58.4)$ & 0.0001 \\
\hline$D_{\text {mean }}$ (non-target breast) (Gy) & $45.5(45.4-45.6)$ & $47.0(38.8-54.3)$ & 0.1590 \\
\hline$D_{1}$ (contralat breast) (Gy) & $0.72(0.4-1.0)$ & $0.64(0.1-1.0)$ & 0.3787 \\
\hline$D_{0.1}$ (contralat breast) (Gy) & $0.99(0.6-1.5)$ & $1.1(0.6-1.6)$ & 0.3341 \\
\hline$D_{1}$ (skin) (Gy) & $58.6(47.2-79.9)$ & $66.7(65.5-67.5)$ & 0.0025 \\
\hline$D_{0.1}$ (skin) (Gy) & $65.8(49.2-85.6)$ & $67.4(65.9-70.4)$ & 0.5197 \\
\hline$D_{1}$ (lung) (Gy) & $32.6(15.7-46.2)$ & $50.6(37.6-64.0)$ & 0.0002 \\
\hline$D_{0.1}$ (lung) (Gy) & $35.3(17.2-48.5)$ & $52.2(38.4-61.7)$ & 0.0002 \\
\hline${ }^{*} D_{1}$ (heart) (Gy) & $50.6(37.6-61.7)$ & $53.2(51.0-55.5)$ & 0.0765 \\
\hline${ }^{*} D_{0.1}$ (heart) (Gy) & $52.2(38.4-64.0)$ & $58.1(51.1-69.1)$ & 0.0009 \\
\hline$D_{1}$ (ribs) (Gy) & $40.2(34.1-48.1)$ & $35.0(20.0-57.3)$ & 0.0642 \\
\hline$D_{0.1}$ (ribs) (Gy) & $44.3(40.0-53.0)$ & $37.7(26.6-60.5)$ & 0.0062
\end{tabular}

$D_{90}$-the minimum dose delivered to $90 \%$ of PTV boost (Gy), $D_{\text {mean }}$ (non-target breast) - the mean dose of non-target breast, $D_{1}(x), D_{0.1}(x)$ - the minimal dose of the most exposed 1 and $0.1 \mathrm{~cm}^{3}$ of ' $x$ ' organ at risk, where $x$ are contralateral breast (contralat breast), skin, lung, heart, and ribs, *left-sided tumors, ${ }^{* *}$ Wilcoxon-matched pairs test 
Table 2. $\mathrm{EQD}_{2}$ total doses of external beam radiation therapy plus interstitial HDR-BT boost calculated by our biological dose summation (BDS) and the uniform dose conception (UDC) method

\begin{tabular}{lccc}
$\mathrm{EQD}_{2}$ & BDS & UDC & $p$-value* \\
\hline $\mathrm{D}_{90}$ (Gy) & $67.1(64.9-73.7)$ & $66.6(65.3-72.2)$ & $\mathbf{0 . 0 3 8 6}$ \\
\hline $\mathrm{D}_{\text {mean }}$ (non-target breast) (Gy) & $45.5(45.4-45.6)$ & $45.5(45.5-45.6)$ & 0.7353 \\
\hline $\mathrm{D}_{1}$ (skin) (Gy) & $58.6(47.2-79.9)$ & $57.7(47.2-73.5)$ & 0.3061 \\
\hline$D_{0.1}$ (skin) (Gy) & $65.8(49.2-85.6)$ & $63.5(46.2-88.4)$ & 0.0534 \\
\hline$D_{1}$ (lung) (Gy) & $32.6(15.7-46.2)$ & $50.1(47.0-57.3)$ & $\mathbf{0 . 0 0 0 1}$ \\
\hline$D_{0.1}$ (lung) (Gy) & $35.3(17.2-48.5)$ & $51.1(47.2-60.3)$ & $\mathbf{0 . 0 0 0 1}$ \\
\hline$D_{1}$ (ribs) (Gy) & $40.2(34.1-48.1)$ & $51.4(47.0-61.6)$ & $\mathbf{0 . 0 0 0 1}$ \\
\hline$D_{0.1}$ (ribs) (Gy) & $44.3(40.0-53.0)$ & $53.5(47.5-65.7)$ & $\mathbf{0 . 0 0 0 3}$
\end{tabular}

$D_{90}$ - the minimum dose delivered to $90 \%$ of PTV boost (Gy), $D_{\text {mean }}$ (non-target breast) - the mean dose of non-target breast, $D_{1}(x), D_{0.1}(x)$ - the minimal dose of the most exposed 1 and $0.1 \mathrm{~cm}^{3}$ of ' $x$ ' organ at risk, where $x$ are skin, lung, and ribs, *Wilcoxon-matched pairs test

PTV, the ratio of PTV and the whole breast, the volume irradiated of at least prescribed dose, the number of catheters, and TRAK increased the risk of late side effects. The volume irradiated at least $150 \%$ of prescribed dose causes more grade 1 pain in the breast, while maximal dose of the skin increases the risk of grade 1 hyperpigmentation. The $\mathrm{EQD}_{2}$ prescribed dose to the PTV boost with our fractionation scheme was 65.3 Gy using BT and 56.4 Gy with EBRT boost. Despite the fact that BT irradiated the boost volume with almost $10 \mathrm{~Gy}$ additional dose than EBRT boost technique, at the same time, the dose to OARs was reduced with BT. In our study, using EBRT with HDR-BT boost doses to all OARs could be kept under tolerance levels. The $\mathrm{EQD}_{2} \mathrm{D}_{90}$ of PTV was 67.1 Gy, while the mean dose of non-target breast was $45.5 \mathrm{~Gy}$. The $\mathrm{D}_{1}$ and $\mathrm{D}_{0.1}$ of contralateral breast was negligible, 0.72 and 0.99 Gy. The $\mathrm{D}_{1}$ dose of the skin was $58.6 \mathrm{~Gy}, 87 \%$ of the PTV total dose. The $\mathrm{D}_{1}$ dose to the lung was $32.6 \mathrm{~Gy}$ on average, approximately half of the prescribed dose, while the $\mathrm{D}_{1}$ of the ribs was $40.2 \mathrm{~Gy}$, despite PTV's close proximity to the ribs in cases of deeply seated tumors. The $D_{1}$ to the heart was 50.6 Gy on average, in cases of left sided tumors.

Nevertheless, in EBRT boost, larger target volume was used than in BT, and the total dose to PTV was $18 \%$ less in our patient's cohort; $\mathrm{D}_{90}$ was 67.1 Gy using BT and 56.7 Gy with EBRT boost. There were no significant differences in the dose of non-target and contralateral breast between the two boost techniques. The $\mathrm{D}_{1}$ dose to the skin and lung were smaller, with $14 \%$ (8.1 Gy) and 55\% (18 Gy) of using BT than with EBRT boost. The $\mathrm{D}_{0.1}$ to the heart was slightly higher with EBRT than with BT boost (58.1 Gy vs. $52.2 \mathrm{~Gy})$, but both doses were clinically acceptable. Only dose to the ribs was higher with $\mathrm{BT}$ boost, i.e., the $\mathrm{D}_{1}$ was higher with $15 \%$ (5.2 Gy) than using EBRT boost. It should be noted that no ribs toxicity was detected in our study population. Terheyden et al. [25] concluded the same tendency in case of OARs. They confirmed that there was no difference between BT and EBRT boost for left-sided cancers regarding a dose to the heart, although they used physical maximal point doses in their study. Shahbazian et al. [26] also showed the reduced dose to OARs using BT instead of EBRT boost with photon or electron beams. However, they used only relative dose-volume parameters. The lower dose to critical organs using BT boost can account for less toxicity in BT compared to EBRT boost.

In previous publications, authors used the recommended UDC method to estimate the total dose of prostate and OARs in combined therapy, and calculated relative dose-volume parameters only $[25,26]$. However, they did not consider the real biological doses. Since the most exposed part of the skin, lung, and ribs was in the region where the maximum dose was in BT, and the most exposed part of the contralateral breast and heart was in the region where the maximum dose was in EBRT, this most exposed 1 and $0.1 \mathrm{~cm}^{3}$ could be used for the calculation of total biological dose. In this small volume, we might disregard the quadratic dependence. Therefore, our dose summation method is simple, timesaving, and more personalized than the UDC method. The only more precise method would be a pixel-by-pixel calculation of biological dose in the same organ, after a deformable registration of BT and EBRT image series, but no treatment planning systems provides this possibility at the moment.

The effect of dose summation technique on dose-volume parameters in combined EBRT and BT was also investigated in our study. The EQD $\mathrm{D}_{90}$ of PTV boost was $0.7 \%$ higher in our BDS than in the conventional UDC method, but this 0.5 Gy difference was clinically negligible. The mean dose to the non-target breast and $D_{1}$ to skin was practically equivalent in our BDS and the UDC method. Nevertheless, the UDC overestimated the total $\mathrm{D}_{1}$ dose to lung by $54 \%(17.5 \mathrm{~Gy})$ and $\mathrm{D}_{1}$ dose to ribs by $2.5 \%$ (11.2 Gy) compared to BDS method. The cause may be the development of EBRT techniques, such as using field-in-field technique instead of wedges and image guidance during dose delivery, resulting in decreased dose of critical structures. Accordingly, the potential advantage of the BDS method is that it considers the most exposed part of OARs and thus, sparing these parts from higher doses in EBRT before boost irradiation. Overall, 
the dose to the OARs can be reduced using our alternative dose summation method, therefore the treatmentrelated toxicity can be decreased.

It has to be mentioned that this dose summation method can cause some uncertainties. Possible sources of error could include subjectivity of the manual registration process, difference between the EBRT and BT CTV boost, and possible movement of the surgical clips in the tumor bed due to tissue necrosis.

This study is the starting point of the development of an algorithm for the summation of EBRT and BT biologically effective doses, which uses an artificial-intelligencebased DIR algorithm to match the critical anatomical structures in two radiotherapy modalities. Further investigations are needed to assess whether our method predicts toxicity better than the recent UDC method.

\section{Conclusions}

Based on our biological dose summation method in EBRT for whole breast with interstitial HDR-BT or EBRT boost treatment in early-stage breast cancer, the total dose of PTV boost is higher using BT boost than EBRT. Following the recommended fractionation scheme, BT boost yields lower skin, lung, and heart doses, but higher dose to ribs. UDC overestimates lung and ribs doses compared to our method.

\section{Disclosure}

This study was supported by the János Bolyai Research Scholarship of the Hungarian Academy of Sciences and the ÚNKP-18-4 New National Excellence Program of the Ministry of Human Capacities.

The authors report no conflict of interest.

\section{References}

1. Fisher B, Anderson S, Bryant J et al. Twenty-year follow-up of a randomized trial comparing total mastectomy, lumpectomy, and lumpectomy plus irradiation for the treatment of invasive breast cancer. N Engl J Med 2002; 347: 1233-1241.

2. Veronesi U, Cascinelli N, Mariani L et al. Twenty-year follow-up of a randomized study comparing breast-conserving surgery with radical mastectomy for early breast cancer. N Engl J Med 2002; 347: 1227-1232.

3. NIH Consensus Conference. Treatment of early-stage breast cancer. JAMA 1991; 265: 391-395.

4. Polgár C, Fodor J, Major T et al. The role of boost irradiation in the conservative treatment of stage I-II breast cancer. Pathol Oncol Res 2001; 7: 241-250.

5. Bartelink H, Horiot JC, Poortmans $\mathrm{H}$ et al. Impact of a higher radiation dose on local control and survival in breast-conserving therapy of early breast cancer: 10 -year results of the randomized boost versus no boost EORTC 22881-10882 trial. J Clin Oncol 2007; 25: 3259-3265.

6. Polgár C, Fodor J, Orosz Z et al. Electron and high dose rate brachytherapy boost in the conservative treatment of stage I-II breast cancer: First results of the randomized Budapest boost trial. Strahlenther Onkol 2002; 178: 615-623.

7. Romestaing $\mathrm{P}$, Lehingue $\mathrm{Y}$, Carrie $\mathrm{C}$ et al. Role of a 10-Gy boost in the conservative treatment of early breast cancer: Results of a randomized clinical trial in Lyon, France. J Clin Oncol 1997; 15: 963-968.
8. Mansfield CM, Komarnicky LT, Schwartz GF et al. Ten-year results in 1070 patients with stages I and II breast cancer treated by conservative surgery and radiation therapy. Cancer 1995; 75: 2328-2336.

9. Polgár C, Major T. Current status and perspectives of brachytherapy for breast cancer. Int J Clin Oncol 2009; 14: 7-24.

10. Touboul E, Belkacemi Y, Lefranc JP et al. Early breast cancer: influence of type of boost (electron vs iridium-192 implant) on local control and cosmesis after conservative surgery and radiation therapy. Radiother Oncol 1995; 34: 105-113.

11. Vicini FA, Horwitz EM, Lacerna MD et al. Long-term outcome with interstitial brachytherapy in the management of patients with early-stage breast cancer treated with breast-conserving therapy. Int J Radiat Oncol Biol Phys 1997; 37: 845-852.

12. Wazer DE, Kramer B, Schmid C et al. Factors determining outcome in patients treated with interstitial implantation as a radiation boost for breast conservation therapy. Int J Radiat Oncol Biol Phys 1997; 39: 381-393.

13. Resch A, Pötter R, Van Limbergen E et al. Long-term results (10 years) of intensive breast conserving therapy including a high-dose and large-volume interstitial brachytherapy boost (LDR/PDR) for T1/T2 breast cancer. Radiother Oncol 2002; 63: 47-58

14. Budrukkar AN, Sarin R, Shrivastava SK et al. Cosmesis, late sequelae and local control after breast-conserving therapy: influence of type of tumour bed boost and adjuvant chemotherapy. Clin Oncol 2007; 19: 596-603.

15. Fijuth J. Brachytherapy in breast cancer. J Contemp Brachytherapy 2009; 1: 117-120.

16. Guinot JL, Roldan S, Maronas M et al. Breast-conservative surgery with close or positive margins: can the breast be preserved with high-dose-rate brachytherapy boost? Int J Radiat Oncol Biol Phys 2007; 68: 1381-1387.

17. Kauer-Dorner D, Berger D. The role of brachytherapy in the treatment of breast cancer. Breast Care 2018; 13: 157-161.

18. Poortmans P, Bartelink H, Horiot JC et al. The influence of the boost technique on local control in breast conserving treatment in the EORTC boost versus no boost randomised trial. Radiother Oncol 2004; 72: 25-33.

19. Polgár C, Jánváry L, Major T et al. The role of high-dose-rate brachytherapy boost in breast-conserving therapy: Longterm results of the Hungarian National Institute of Oncology. Rep Pract Oncol Radiother 2010; 15: 1-7.

20. Fröhlich G, Major T, Polgár C. Evaluation of the dosimetric parameters and the late side effects in CT-guided partial breast brachytherapy (in Hungarian). Magy Onkol 2011; 55: 132.

21. Fowler JF. The linear-quadratic formula on progress in fractionated radiotherapy. Br J Radiol 1989; 62: 679-694.

22. Nag S, Gupta N. A simple method of obtaining equivalent doses for use in HDR brachytherapy. Int J Radial Oncol Biol Phys 2000; 46: 507-513.

23. Haie-Meder C, Pötter R, Van Limbergen E et al. Recommendations from Gynaecological (GYN) GEC-ESTRO Working Group (I): Concepts and terms in 3D image based 3D treatment planning in cervix cancer brachytherapy with emphasis on MRI assessment on GTV and CTV. Radiother Oncol 2005; 74: $235-245$

24. Fröhlich G, Lang S, Berger D et al. Spatial relationship of the 3D dose distribution from brachytherapy and external beam therapy for adding both dose plans in patients with cervix cancer. Brachytherapy 2008; 7: 95.

25. Terheyden MM, Melchert C, Kovács G. External beam boost versus interstitial high-dose-rate brachytherapy boost in the adjuvant radiotherapy following breast-conserving therapy in early-stage breast cancer: a dosimetric comparison. J Contemp Brachytherapy 2016; 8: 294-300. 
26. Shahbazian H, Bakhshali R, Shamsi A, Bagheri A. Dosimetric analysis of breast cancer tumor bed boost: An interstitial brachytherapy vs. external beam radiation therapy comparison for deeply seated tumors. Brachytherapy 2020; 19: 264-274.

27. Fröhlich G, Vízkeleti J, Nguyen Anhhong N et al. Comparative analysis of image-guided adaptive interstitial brachytherapy and intensity-modulated arc therapy versus conventional treatment techniques in cervix cancer using biological dose summation. J Contemp Brachytherapy 2019; 11: 69-75

28. Fröhlich G, Ágoston P, Jorgo K et al. Biological dose summation of intensity-modulated arc therapy and image-guided high-dose-rate interstitial brachytherapy in intermediate and high-risk prostate cancer. J Contemp Brachytherapy 2020; 12 260-266.

29. Haviland JS, Owen JR, Dewar JA et al. The UK Standardisation of Breast Radiotherapy (START) trials of radiotherapy hypofractionation for treatment of early breast cancer: 10-year follow-up results of two randomised controlled trials. Lancet Oncol 2013; 14: 1086-1094.

30. Major T, Fröhlich G, Lövey K et al. Dosimetric experience with accelerated partial breast irradiation using image-guided interstitial brachytherapy. Radiother Oncol 2009; 90: 48-55.

31. Major T, Polgár Cs, Fröhlich G. Dosimetric characteristics of accelerated partial breast irradiation with $\mathrm{CT}$ image-based multi-catheter interstitial brachytherapy: A single institution's experience. Brachytherapy 2011; 10: 421-426.

32. Major T, Polgár C, Fröhlich G. Assessment of dose homogeneity in conformal interstitial breast brachytherapy with special respect to ICRU recommendations. J Contemp Brachytherapy 2011; 3: 150-155.

33. Fröhlich G, Geszti Gy, Vízkeleti J et al. Dosimetric comparison of inverse optimisation methods versus forward optimisation in HDR brachytherapy of breast, cervix and prostate cancer. Strahlenther Onkol 2019; 195: 991-1000.

34. Major T, Fröhlich G, Mészáros N et al. Does the inverse planning improve the plan quality in interstitial high dose rate breast brachytherapy? J Contemp Brachytherapy 2020; 12: $166-174$ 